\title{
ABSENCE OF GEODESICS IN FIRST-PASSAGE PERCOLATION ON A HALF-PLANE
}

\author{
By J AN WEHR AND J UNG WOO \\ University of Arizona
}

\begin{abstract}
An H-geodesic is a doubly infinite path which locally minimizes the passage time in the i.i.d. first passage percolation model on a half-plane $\mathbf{H}$. Under the assumption that the bond passage times are continuously distributed with a finite mean, we prove that, with probability $1, \mathbf{H}$-geodesics do not exist. As a corollary we show that, with probability 1, any geodesic in the analogous model on the whole plane $\mathbf{Z}^{2}$ has to intersect all straight lines with rational slopes.
\end{abstract}

1. Introduction. First passage percolation has been a subject of intense research in recent years. References [1] and [6] are excellent general references which also include big bibliographies. Among other recent papers on first passage percolation of general interest, we mention [3]. Below we define the model and state our main results as well as make some remarks about their relation to other work.

The subset of $\mathbf{R}^{2}$ is $\mathbf{Z}^{2}$, consisting of the points with integer coordinates. Elements of $\mathbf{Z}^{2}$ are called sites. $A$ bond of $\mathbf{Z}^{2}$ is a line segment connecting two sites with Euclidean distance 1 . If $\mathbf{L}$ is a subset of $\mathbf{Z}^{2}$, a bond of $\mathbf{L}$ is a bond of $\mathbf{Z}^{2}$, both of whose endpoints are in $\mathbf{L}$.

A finite path is an alternating sequence $\left(x_{0}, b_{1}, x_{1}, b_{2}, \ldots, x_{n-1}, b_{n}, x_{n}\right)$ of sites and bonds of $\mathbf{Z}^{2}$ such that for $i=1, \ldots, n, b_{i}$ is the bond connecting $x_{i-1}$ to $x_{i}$ (and, in particular, the distance from $x_{i-1}$ to $x_{i}$ equals one). An infinite path is defined similarly, with i ranging through all integers (positive, negative and zero). Note that with this definition, an infinite path is infinite "in both directions," that is, it has no beginning and no end. A path in $\mathbf{L}$ is a path all of whose sites and bonds are sites and bonds of $\mathbf{L}$. A path can naturally be considered as a subset of $\mathbf{R}^{2}$ and we will sometimes use the word "path" in this sense. For path $\gamma$ and $\mathrm{x}$ and $\mathrm{y}$ sites of $\gamma, \gamma_{\mathrm{x}, \mathrm{y}}$ is called a segment of $\gamma$ if it is a path with two end sites $x$ and $y$ and it is a subset of $\gamma$. We say that two paths $\gamma$ and $\gamma^{\prime}$ meet more than once if there exist $\mathrm{x}, \mathrm{y} \in \gamma \cap \gamma^{\prime}$ such that $\gamma_{\mathrm{x}, \mathrm{y}} \neq \gamma_{\mathrm{x}, \mathrm{y}}^{\prime}$.

Let $\left\{t_{b}: b\right.$ is a bond in $\left.\mathbf{z}^{2}\right\}$ be a family of independent identically distributed (shortly i.i.d.) continuous positive real-valued random variables on a

Received October 1996; revised April 1997.

AMS 1991 subject classifications. 60K 35, 82B44, 82D30.

Key words and phrases. First-passage percolation, time-minimizing paths, infinite geodesics, ergodicity, large deviation bounds. 
probability space $(\Omega, \mathbf{P})$. For a finite path $\gamma$, we define the passage time of $\gamma$,

$$
\mathrm{t}(\gamma)=\sum_{i=1}^{\mathrm{n}} \mathrm{t}_{\mathrm{b}_{\mathrm{i}}}
$$

where $b_{i}$ runs through all bonds of $\gamma$. For a given connected subset $\mathbf{L}$, a first passage time in $\mathbf{L}$ between two sites $x$ and $y$ of $\mathbf{L}$ [denoted $t_{\mathbf{L}}(x, y)$ ] is defined as the infimum of passage times of all paths in $\mathbf{L}$ with two end sites $x$ and $y$. A path which achieves the infimum always exists and is unique (see Lemma 8 below). It is called the finite $\mathbf{L}$-geodesic connecting $x$ to $y$ and denoted $\gamma_{\mathbf{L}}(\mathbf{x}, \mathbf{y})$. Thus a finite $\mathbf{L}$-geodesic minimizes the passage time in $\mathbf{L}$ between its two end sites in $\mathbf{L}$, that is,

$$
t\left(\gamma_{\mathbf{L}}(x, y)\right)=t_{L}(x, y)
$$

Note that any segment of $\gamma_{\mathbf{L}}(\mathrm{x}, \mathrm{y})$ is also a finite $\mathbf{L}$-geodesic. We now define what it means for an infinite path in $\mathbf{L}$ to be an $\mathbf{L}$-geodesic. This is the main object studied in this work.

DEFINITION 1. An infinite path $\gamma$ in $\mathbf{L}$ is called an infinite $\mathbf{L}$-geodesic (or simply an $\mathbf{L}$-geodesic) if all its segments $\gamma_{x, y}$ are finite $\mathbf{L}$-geodesics. $\mathbf{Z}^{2}$-geodesics will simply be called geodesics.

While finite geodesics always exist, existence of infinite geodesics is a totally different matter. This question was, to the best of our knowledge, first asked by $\mathrm{H}$. Furstenberg (see [1]). It is equivalent to the problem of existence of nontrivial (i.e., nonconstant) ground states in the two-dimensional Ising ferromagnet with random exchange constants (see the forthcoming lecture notes [6] and references therein). Ground states of higher-dimensional random ferromagnets are similarly related to hypersurfaces with minimal random weights ([4], [6]). It is believed that on $\mathbf{Z}^{2}$ infinite geodesics do not exist. Partial results in this direction have been obtained in [5] and [7] (see also [6]). It is proven there that, with the unverified uniform curvature hypothesis of first-passage asymptotic shape, there cannot exist any geodesics other than those with asymptotic directions $\hat{x}$ and $\hat{y}$ at both infinities [such geodesics are called $(\hat{x}, \hat{y})$ geodesics there]. Furthermore, $(\hat{x}, \hat{y})$ geodesics were ruled out for almost all ( $\hat{x}$ and $\hat{y}$ ). In [8] it is shown that the number of geodesics is either zero or infinity. This last result holds in all dimensions and applies also to the case of minimal hypersurfaces. It is possible that the technique of the present paper can also be generalized to minimal hypersurfaces in higher dimensions.

The MAIN ASSUMPTION. Throughout this paper we will assume that $t_{b}$ are i.i.d. positive real-valued random variables with a continuous distribution such that $\mathbf{E}\left[t_{b}\right]<\infty$. It guarantees the following facts, which we will use in 
the proof:

No two different L-geodesics (finite or infinite) meet more than once.

Any event invariant under translation by some integer lattice vector has probability zero or one.

A large deviation estimate for the number of bonds of a finite L-geodesic holds (see Lemma 9).

We now state our main results: let $\mathbf{H}$ denote the upper half-plane

$$
\mathbf{H}=\left\{\left(\mathrm{x}_{1}, \mathrm{x}_{2}\right): \mathrm{x}_{2} \geq 0\right\} \text {. }
$$

TheOREM 2. With probability 1 there exists no $\mathbf{H}$-geodesic.

It follows from the above theorem that with probability 1 any geodesic (i.e., $\mathbf{Z}^{2}$-geodesic) has to intersect the horizontal coordinate axis. Moreover, given any straight line, with probability 1 any geodesic intersects this line. Hence the following corollary (for a more detailed proof, see the end of the next section).

COROLLARY 3. Given any straight linel in $\mathbf{R}^{2}$, consider a half-plane whose boundary is I. We denote the intersection of this half-plane with the lattice $\mathbf{Z}^{2}$ by $\mathbf{H}_{1}$. Then, with probability 1 there is no $\mathbf{H}_{1}$-geodesic. As a consequence, with probability 1 all $\mathbf{Z}^{2}$-geodesics (if they exist) have to intersect all straight lines with rational slopes.

2. Proof of main results. Let us introduce an event

$$
\mathrm{K}^{*}=\{\text { there exists at least one } \mathbf{H} \text {-geodesic }\} \text {. }
$$

Since $\mathrm{K}^{*}$ is translation invariant under horizontal translations, by (2) $\mathrm{K} *$ is a zero or one event. Hence in order to prove the main theorem, we only need to rule out $\mathrm{P}\left[\mathrm{K}^{*}\right]=1$. Therefore we will assume $\mathrm{P}\left[\mathrm{K}^{*}\right]=1$ throughout this section and reach two contradictory statements [(23) and (24)], which will imply Theorem 2.

Note that any $\mathbf{H}$-geodesic $\gamma$ divides $\mathbf{R}^{2} \backslash \gamma$ into two components, say, $\mathrm{R}^{+}=\mathrm{R}^{+}(\gamma)$, and $\mathrm{R}^{-}=\mathrm{R}^{-}(\gamma)$, that is,

$$
\begin{aligned}
\mathrm{R}^{+}(\gamma) \cap \mathrm{R}^{-}(\gamma) & =\varnothing, \\
\mathrm{R}^{+}(\gamma) \cup \mathrm{R}^{-}(\gamma) & =\mathbf{R}^{2} \backslash \gamma, \\
\partial \mathrm{R}^{+} & =\partial \mathrm{R}^{-}=\gamma,
\end{aligned}
$$

where $\mathrm{R}^{-}$is a region that contains $(0,-1)$ and where $\partial \mathrm{A}$ denotes the usual boundary of a set $A$ in $\mathbf{R}^{2}$. Hence for any points $x, y \in R^{-}(\gamma)$, no bond $b$ belonging to the finite $\mathbf{H}$-geodesic $\gamma_{\mathbf{H}}(\mathrm{x}, \mathrm{y})$ can be an element of $\mathbf{R}^{+}(\gamma)$, because otherwise two different finite $\mathbf{H}$-geodesics would meet more than once. Now we are ready to prove the following proposition. 
Proposition 4. Assume $\mathbf{P}\left[K^{*}\right]=1$. Consider the sequence of finite $\mathbf{H}$-geodesics $\gamma_{\mathbf{H}}\left(x_{n}, y_{n}\right)$, where $x_{n}=(-n, 0), y_{n}=(n, 0), n \in \mathbf{Z}_{+}$. With probability 1 , this sequence has a limit:

$$
\gamma_{0}=\lim _{n \rightarrow \infty} \gamma_{\mathbf{H}}\left(x_{n}, y_{n}\right)
$$

Moreover, $\gamma_{0}$ is an $\mathbf{H}$-geodesic and for any $\mathbf{H}$-geodesic $\gamma$,

$$
\gamma_{0} \subset \mathrm{R}^{-}(\gamma) \cup \gamma .
$$

The last statement says that $\gamma_{0}$ lies on or bel ow any other $\mathbf{H}$-geodesic.

Proof. Let us introduce rectangular boxes and events which are used in the rest of this section:

$$
\begin{aligned}
& \mathrm{B}=\mathrm{B}(\mathrm{I})=\left\{\left(\mathrm{x}_{1}, \mathrm{x}_{2}\right) \in \mathbf{Z}^{2}:-\mathrm{I} \leq \mathrm{x}_{1} \leq \mathrm{I}, 0 \leq \mathrm{x}_{2} \leq 2 \mathrm{I}\right\}, \\
& \mathrm{K}=\mathrm{K}(\mathrm{I})=\{\text { At least one } \mathbf{H} \text {-geodesic intersects } \mathrm{B}(\mathrm{I})\},
\end{aligned}
$$

where I is a positive integer. Take $\omega \in \mathrm{K}^{*}$. Choose any $\mathbf{H}$-geodesic $\gamma$, and pick I such that

$$
\mathrm{B}(\mathrm{I}) \cap \gamma \neq \varnothing \text {. }
$$

Note that

$$
\gamma_{\mathbf{H}}\left(\mathrm{x}_{\mathrm{n}}, \mathrm{y}_{\mathrm{n}}\right) \text { is a subset of } \mathrm{R}^{-}(\gamma) \cup \gamma,
$$

since otherwise two $\mathbf{H}$-geodesics $\gamma$ and $\gamma_{\mathbf{H}}\left(\mathbf{x}_{n}, \mathbf{y}_{n}\right)$ would meet more than once. It follows from (6) and (7) that all $\gamma_{\mathbf{H}}\left(x_{n}, y_{n}\right)$ intersect $B(I)$. Hence there exists $a$ bond $b$ in $B(I)$ and a subsequence of $\gamma_{H}\left(x_{n}, y_{n}\right)$, all elements of which contain $b$. Continuing this procedure for consecutive bonds and choosing further subsequences, by a standard diagonal argument there exist a doubly infinite path $\gamma_{0}$ and a subsequence of finite $\mathbf{H}$-geodesics $\gamma_{\mathbf{H}}\left(\mathrm{x}_{\mathrm{n}_{\mathrm{k}}}, \mathrm{y}_{\mathrm{n}_{\mathrm{k}}}\right)$, which contain any fixed bond of $\gamma_{0}$ for all sufficiently large $\mathrm{k}$. By construction of $\gamma_{0}$ any segment of $\gamma_{0}$ is a subset of $\gamma_{\mathbf{H}}\left(\mathbf{x}_{\mathrm{n}_{\mathrm{k}}}, \mathrm{y}_{\mathrm{n}_{\mathrm{k}}}\right)$ for some $\mathrm{k}$, which implies that any segment of $\gamma_{0}$ is a finite $\mathbf{H}$-geodesic. Hence $\gamma_{0}$ is an infinite $\mathbf{H}$-geodesic. Since the sequence $\gamma_{\mathbf{H}}\left(x_{n}, y_{n}\right)$ is monotone in the sense that for $\mathrm{n}>\mathrm{m}, \gamma_{\mathbf{H}}\left(\mathrm{x}_{\mathrm{n}}, \mathrm{y}_{\mathrm{n}}\right)$ lies above or on $\gamma_{\mathbf{H}}\left(\mathrm{x}_{\mathrm{m}}, \mathrm{y}_{\mathrm{m}}\right)$, it now follows that the whole sequence converges to $\gamma_{0}$ and from (7) it follows that $\gamma_{0}$ lies on or below any H-geodesic.

Lemma 5. Let $\mathrm{n}$ be a positiveinteger and let $\mathbf{H}^{\prime}=\left\{\left(\mathrm{x}_{1}, \mathrm{x}_{2}\right) \in \mathbf{R}^{2}: \mathrm{x}_{2} \leq \mathrm{n}\right\}$. With probability 1 , for any infinite $\mathbf{H}$-geodesic $\gamma$ intersecting the site $z=$ $\left(z_{1}, z_{2}\right)$ with $z_{2}<n$,

$$
\mathbf{H}^{\prime} \cap \mathbf{R}^{+}(\gamma) \text { is nonempty and all its components are bounded. }
$$

Boundary of each component is a self-avoiding loop, which is a bond-disjoint union of segments of $\gamma$ and segments of the boundary of $\mathbf{H}^{\prime}$. 
Proof. In order to prove the boundedness of each component of $\mathbf{H}^{\prime} \cap$ $\mathrm{R}^{+}(\gamma)$, it is sufficient to prove that

$$
\begin{aligned}
& \mathbf{P}[\text { There exists an } \mathbf{H} \text {-geodesic with an infinite connected } \\
& \text { part contained in } \left.\mathbf{H}^{\prime}\right]=0 \text {. }
\end{aligned}
$$

Since the distribution of $t_{b}$ is continuous, for some $0<\alpha<\beta$ we have $\mathbf{P}\left[\mathrm{t}_{\mathrm{b}}<\alpha\right]>0$ and $\mathbf{P}\left[\mathrm{t}_{\mathrm{b}}>\beta\right]>0$. Take a positive integer $\mathrm{m}>2 \mathrm{n} \alpha /(\beta-\alpha)$. Then

$$
\beta \mathrm{m}>\alpha \times(\mathrm{m}+2 \mathrm{n}) .
$$

For each integer $k$, consider a rectangular box

$$
\mathrm{C}_{\mathrm{k}}=\mathrm{C}_{\mathrm{k}}(\mathrm{m}, \mathrm{n})=\left\{\left(\mathrm{x}_{1}, \mathrm{x}_{2}\right): 2 \mathrm{~km} \leq \mathrm{x}_{1} \leq(2 \mathrm{k}+1) \mathrm{m}, 0 \leq \mathrm{x}_{2} \leq \mathrm{n}\right\} \text {. }
$$

We say that a path in $\mathbf{H}$ crosses $C_{k}$ if its segment connects the left and the right boundaries of $C_{k}$ inside $C_{k}$, without intersecting the upper boundary of $\mathrm{C}_{\mathrm{k}}$. Consider the events

$$
\begin{aligned}
& F_{k}=\left\{\text { At least one } \mathbf{H} \text {-geodesic crosses } C_{k}\right\}, \\
& E_{k}=\left\{t_{b}<\alpha \text { for all bonds } b \in \partial C_{k} \cap\left\{x_{2}>0\right\}\right. \text { and } \\
& \left.\qquad t_{b}>\beta \text { for all other bonds } b \in C_{k}\right\} .
\end{aligned}
$$

Note that $E_{k}$ are independent with the same positive probability and that for the choice of $m$ in (10) and for each $k, E_{k}$ is disjoint with $F_{k}$. By the Borel-Cantelli lemma

$$
\mathbf{P}\left[\mathrm{E}_{\mathrm{k}} \text { holds for infinitely many positive } \mathrm{k}\right]=1 \text {. }
$$

Hence we get

$$
\mathbf{P}\left[\mathrm{F}_{\mathrm{k}} \text { holds for all sufficiently large } \mathrm{k}\right]=0 \text {. }
$$

Similarly we get

$$
\mathbf{P}\left[\mathrm{F}_{\mathrm{k}} \text { holds for all sufficiently small } \mathrm{k}\right]=0 \text {. }
$$

From (12) and (13), we obtain (9). The rest of the lemma follows immediately.

Let us recall that $\mathrm{B}=\mathrm{B}(\mathrm{I})=\left\{\left(\mathrm{x}_{1}, \mathrm{x}_{2}\right) \in \mathrm{Z}^{2}\right.$ : $\left.-\mathrm{I} \leq \mathrm{x}_{1} \leq \mathrm{I}, 0 \leq \mathrm{x}_{2} \leq 2 \mathrm{I}\right\}$ and that $K$ is the event that at least one $\mathbf{H}$-geodesic intersects $\mathrm{B}(\mathrm{I})$. Define translations of $\mathrm{B}=\mathrm{B}(\mathrm{I})$ for $0 \leq \mathrm{i}, \mathrm{j}<\mathrm{k}$ and $\mathrm{L}>0$,

$$
B_{i, j}=B_{i, j}(I, L)=B+(i L, j L) \text {. }
$$

Note that $B_{i, j}$ are mutually disjoint for $L>2$ I.

Proposition 6. Let

$$
\delta=1-\mathbf{P}[\mathrm{K}] .
$$

$$
\begin{aligned}
\mathbf{P}\left[\mathrm{B}_{\mathrm{i}, \mathrm{j}} \subset \mathrm{R}^{+}\left(\gamma_{0}\right)\right] & \leq \delta, \\
\mathbf{P}\left[\mathrm{B}_{\mathrm{i}, \mathrm{j}} \subset \mathrm{R}^{-}\left(\gamma_{0}\right)\right] & \leq \delta, \\
\mathbf{P}\left[\gamma_{0} \cap \mathrm{B}_{\mathrm{i}, \mathrm{j}} \neq \varnothing \text { for all } 0 \leq \mathrm{i}, \mathrm{j}<\mathrm{k}\right] & \geq 1-2 \mathrm{k}^{2} \delta .
\end{aligned}
$$


Proof. Let

$$
\begin{aligned}
\mathbf{H}_{\mathrm{j}} & =\left\{\left(\mathrm{x}_{1}, \mathrm{x}_{2}\right): \mathrm{x}_{2} \geq \mathrm{jL}\right\}, \\
\mathbf{H}_{\mathrm{j}}^{\text {low }} & =\left\{\left(\mathrm{x}_{1}, \mathrm{x}_{2}\right): \mathrm{x}_{2} \leq \mathrm{jL}+2 \mathrm{I}\right\} .
\end{aligned}
$$

Define the following events:

$$
\begin{aligned}
\mathrm{K}_{\mathrm{i}, \mathrm{j}} & =\left\{\text { At least one } \mathbf{H}_{\mathrm{j}} \text {-geodesic intersects } \mathrm{B}_{\mathrm{i}, \mathrm{j}}\right\}, \\
\mathrm{K}_{\mathrm{i}, \mathrm{j}}^{\text {low }} & =\left\{\text { At least one } \mathbf{H}_{\mathrm{j}}^{\text {low }} \text {-geodesic intersects } \mathrm{B}_{\mathrm{i}, \mathrm{j}}\right\} .
\end{aligned}
$$

Obviously the above events and sets depend on I and L. Since I and L remain fixed throughout the proof, we will suppress this dependence in the notation. Since our model is invariant under translations by integer vectors and under rotations by $180^{\circ}$, we have

$$
\mathbf{P}[\mathrm{K}]=\mathbf{P}\left[\mathrm{K}_{\mathrm{i}, \mathrm{j}}\right]=\mathbf{P}\left[\mathrm{K}_{\mathrm{i}, \mathrm{j}}^{\text {low }}\right] \text {. }
$$

Note that

$$
\mathrm{K}=\left\{\gamma_{0} \text { intersects } \mathrm{B}\right\} .
$$

Let us introduce the following events:

$$
E_{i, j}=\left\{\gamma_{0} \cap B_{i, j} \neq \varnothing\right\} .
$$

For each pair $(i, j), K^{*}$ is a disjoint union of $\left\{B_{i, j} \subset R^{+}\left(\gamma_{0}\right)\right\},\left\{B_{i, j} \subset R^{-}\left(\gamma_{0}\right)\right\}$ and $E_{i, j}$, that is,

$$
\mathrm{K}^{*}=\left\{\mathrm{B}_{\mathrm{i}, \mathrm{j}} \subset \mathrm{R}^{+}\left(\gamma_{0}\right)\right\} \cup\left\{\mathrm{B}_{\mathrm{i}, \mathrm{j}} \subset \mathrm{R}^{-}\left(\gamma_{0}\right)\right\} \cup \mathrm{E}_{\mathrm{i}, \mathrm{j}} .
$$

First we will prove (15). Assume $\mathbf{B}_{i, j} \subset \mathbf{R}^{+}\left(\gamma_{0}\right)$. Since the set $\mathbf{R}^{+}\left(\gamma_{0}\right) \cap \mathbf{H}_{j}^{\text {low }}$ contains $B_{i, j}$, by Lemma 5 exactly one of its components (bounded) contains $B_{i, j}$. Hence there is no $\mathbf{H}_{j}^{\text {low }}$-geodesic intersecting $B_{i, j}$ since otherwise two different finite $\mathbf{H}_{\mathrm{j}}^{\text {low }} \cap \mathbf{H}$-geodesics would meet more than once [see (2)]. Therefore,

$$
\left\{\mathrm{B}_{\mathrm{i}, \mathrm{j}} \subset \mathrm{R}^{+}\left(\gamma_{0}\right)\right\} \subset \Omega \backslash \mathrm{K}_{\mathrm{i}, \mathrm{j}}^{\text {low }} .
$$

Using (14), (18) and (20), we get (15).

To prove (16), assume $\mathrm{B}_{\mathrm{i}, j} \subset \mathrm{R}^{-}\left(\gamma_{0}\right)$. By monotone convergence of $\gamma_{\mathbf{H}}\left(x_{n}, y_{n}\right)$ to $\gamma_{0}$ (see Proposition 4), for sufficiently large $n, B_{i, j}$ lies inside the loop $\gamma_{\mathbf{H}}\left(x_{n}, y_{n}\right) \cup\left[x_{n}, y_{n}\right]$, where $\left[x_{n}, y_{n}\right]$ is the horizontal line segment connecting $x_{n}=(-n, 0)$ to $y_{n}=(n, 0)$. Again by $(2)$, there is no $\mathbf{H}_{j}$-geodesic intersecting $B_{i, j}$, that is,

$$
\left\{B_{i, j} \subset R^{-}\left(\gamma_{0}\right)\right\} \subset \Omega \backslash K_{i, j} .
$$

Using (14), (18) and (21), we end the proof of (16).

To prove (17), from (15), (16) and (19) we have $\mathbf{P}\left[E_{i, j}\right] \geq 1-2 \delta$. Thus for $k>0$,

$$
\mathbf{P}\left[\bigcap_{0 \leq i, j<k} E_{i, j}\right] \geq 1-2 k^{2} \delta .
$$

This completes the proof. 
REMARK. In particular, for any $k>0$, choose I sufficiently large to guarantee $1-\mathbf{P}[K(\mathrm{I})] \leq 1 / 4 \mathrm{k}^{2}$. For such a choice of $\mathrm{I}(\mathrm{k})$ and for any $\mathrm{L},(22)$ implies the following estimate:

$$
\mathbf{P}\left[\bigcap_{0 \leq i, j<k} E_{i, j}(I(k), L)\right] \geq \frac{1}{2} .
$$

Note that the intersection in (23) still depends on $\mathrm{k}$ and $\mathrm{L}$.

The following proposition shows that (23) cannot be true for large $k$ if $L$ is large enough [depending on $\mathrm{k}$ and $\mathrm{I}(\mathrm{k})$ ]. This will be the desired contradiction, which will imply $\mathbf{P}\left[\mathrm{K}^{*}\right]=0$.

Proposition 7. For any $k \geq \max \{8 \mathrm{c}, 2\}$ [ $\mathrm{c}$ is a constant in (33); see Lemma 9 below] and for any $I$, there exists an $L \geq 4$ I such that

$$
\mathbf{P}\left[\bigcap_{0 \leq i, j<k} E_{i, j}(I, L)\right]<\frac{1}{2} \text {. }
$$

Proof. Let us assume $\omega \in \bigcap_{0 \leq i, j<k} E_{i, j}$. Let $L>2 I\left(B_{i, j}\right.$ are mutually disjoint for such $L$ ). Then $\gamma_{0}$ intersects all sets $B_{i, j}$. L et $x_{i, j} \in B_{i, j}$ belong to $\gamma_{0}$ for all pairs $(i, j)$ where $0 \leq \mathrm{i}, \mathrm{j}<\mathrm{k}$. Let $\left\{\mathrm{y}_{\mathrm{m}}: 1 \leq \mathrm{m} \leq \mathrm{k}^{2}\right\}$ denote the points $\left\{x_{i, j}\right\}$ listed in the order in which they occur along $\gamma_{0}$. We have the following decomposition of $\gamma_{0}\left(\mathrm{y}_{1}, \mathrm{y}_{\mathrm{k}^{2}}\right)$ (segment of $\left.\gamma_{0}\right)$ into bond-disjoint pieces:

$$
\gamma_{0}\left(y_{1}, y_{k^{2}}\right)=\bigcup_{1 \leq m \leq k^{2}-1} \gamma_{0}\left(y_{m}, y_{m+1}\right) .
$$

Note that $y_{1}, y_{k^{2}} \in U_{i, j} B_{i, j}$ and, moreover, that $y_{1}$ and $y_{k^{2}}$ belong to different sets $B_{i, j}$, which implies that

$$
\left|\mathrm{y}_{1}-\mathrm{y}_{\mathrm{k}^{2}}\right| \geq \mathrm{L}-2 \mathrm{l} \text {. }
$$

Hence for such $k \geq 2$ and $L \geq 4$,

$$
\left|\gamma_{0}\left(y_{1}, y_{k^{2}}\right)\right| \geq\left(k^{2}-1\right)(L-2 I) \geq \frac{k^{2} L}{4} \text {. }
$$

Therefore from (25) and (26) we get

$$
\bigcap_{0 \leq i, j<k} E_{i, j} \subset \bigcup_{x, y \in U_{i, j} B_{i, j},|x-y| \geq L-2 \mid}\left\{\left|\gamma_{\mathbf{H}}(x, y)\right| \geq \frac{k^{2} L}{4}\right\},
$$

where $\gamma_{\mathbf{H}}(\mathbf{x}, \mathrm{y})$ is a finite $\mathbf{H}$-geodesic connecting $\mathbf{x}$ to $\mathbf{y}$. The $\mathrm{I}^{1}$-diameter of the set $U_{i, j} B_{i, j}$ is less than $2 k L$ and the set $U_{i, j} B_{i, j}$ has exactly $(2 I+1)^{2} k^{2}$ sites. Hence from (27),

$$
\mathbf{P}\left[\bigcap_{0 \leq i, j<k} E_{i, j}\right] \leq\left[(2 \mid+1)^{2} k^{2}\right]^{2} \max _{L-2|\leq| x-y \mid \leq 2 k L} \mathbf{P}\left[\left|\gamma_{\mathbf{H}}(x, y)\right| \geq \frac{k^{2} L}{4}\right]
$$


By taking $k \geq 8 c, \quad a<\left(1 / 4[(2 I+1) k]^{4}\right)$, and $L>\ln 4+4 \ln [(2 I+1) k]$ where $c$, a and $L$ are constants in (33) (see Lemma 9), we get $\left(k^{2} L / 4\right) \geq$ $c|x-y|$ for $x, y \in U_{i, j} B_{i, j}$ and

$$
\begin{aligned}
& \mathbf{P}\left[\bigcap_{0 \leq i, j<k} E_{i, j}\right] \\
& \quad \leq[(2 \mid+1) k]^{4} \max _{L-2 I \leq|x-y| \leq 2 k L} \mathbf{P}\left[\left|\gamma_{\mathbf{H}}(x, y)\right| \geq c|x-y|\right] \\
& \quad \leq[(2 \mid+1) k]^{4}(\exp (-L)+a)<\frac{1}{2}
\end{aligned}
$$

where in (28) we have used Lemma 9. Hence the proof is complete.

Proof of the main theorem. Assuming that $\mathbf{P}\left[\mathrm{K}^{*}\right]=1$, we obtained (23) and (24) which leads to a contradiction. Hence by the zero-onelaw, $\mathbf{P}\left[\mathrm{K}^{*}\right]=0$. This ends the proof of the main theorem.

Proof of CoRollary 3. The proof is very similar to the proof of the main theorem and we will only sketch it. Following the proof of Proposition 4, we construct an $\mathbf{H}_{1}$-geodesic, denoted by $\gamma_{0}\left(\mathbf{H}_{1}\right)$ such that

$$
\gamma_{0}\left(\mathbf{H}_{1}\right) \subset \mathbf{R}^{-}(\gamma) \cup \gamma
$$

for any $\mathbf{H}_{1}$-geodesic $\gamma$. A small modification is necessary in the proof of this new version of Proposition 4: we consider a sequence of finite $\mathbf{H}_{1}$-geodesics $\gamma_{\mathbf{H}_{1}}\left(\mathrm{x}_{n}, \mathrm{y}_{\mathrm{n}}\right)$ where $\mathrm{x}_{\mathrm{n}}$ and $\mathrm{y}_{\mathrm{n}}$ are sequences of points in $\mathbf{H}_{1}$ whose Euclidean distance to $I$ is less than 1 , such that $x_{n}$ and $y_{n}$ go to infinity along $I$ in opposite directions.

Corresponding analogs of Lemma 5, Proposition 6, Proposition 7, (23) and (24) follow immediately, which implies a contradiction. This shows that with probability 1 any $\mathbf{Z}^{2}$-geodesic has to intersect any fixed line I. Using countable additivity of $\mathbf{P}$, the rest of the corollary follows immediately.

3. Proof of the auxiliary lemmas. In this section we prove two auxiliary results used above. While similar results can be found in the existing literature, we state them here in the way suitable for our applications and, for completeness, include short proofs.

Lemma 8. For any connected set $\mathbf{L}$ and sites $x, y$ of $\mathbf{L}$ with probability one there exists a unique finite $\mathbf{L}$-geodesic connecting $x$ to $y$.

Proof. Take any $\varepsilon>0$. Since $\mathbf{L}$ is connected, there exists a path in $\mathbf{L}$, say $\gamma$, connecting $x$ to $y$. Fixing such a path $\gamma$, choose $r>0$ so that

$$
\mathbf{P}[\mathrm{t}(\gamma)<\mathrm{r}]>1-\varepsilon
$$


Since $t_{b}$ is positive we can choose $\delta>0$ such that

$$
\mathbf{P}\left[\mathrm{t}_{\mathrm{b}} \leq \delta\right]<\mathrm{p}_{\mathrm{c}}\left(\mathbf{Z}^{2}\right),
$$

where $p_{c}$ denotes the critical density of bond percolation. We say that a bond $\mathrm{b}$ is $\delta$-open if $\mathrm{t}_{\mathrm{b}} \leq \delta$ and $\delta$-closed otherwise.

Define

$$
\begin{aligned}
M_{S}=\min _{\gamma^{\prime}}\left\{\text { Number of } \delta \text {-closed bonds of } \gamma^{\prime}: \gamma^{\prime}\right. \text { is a path } \\
\text { connecting } \left.x \text { to } \partial B_{s}(x)\right\},
\end{aligned}
$$

where $\partial \mathrm{B}_{\mathrm{s}}(\mathrm{x})$ is the boundary of the rectangle $\mathrm{B}_{\mathrm{s}}(\mathrm{x})=\left\{\mathrm{x}+\left(\mathrm{y}_{1}, \mathrm{y}_{2}\right):\left|\mathrm{y}_{1}\right| \leq\right.$ $\left.\mathrm{s},\left|\mathrm{y}_{2}\right| \leq \mathrm{s}\right\}$. Hence, since by (31) with probability 1 there is no $\delta$-open infinite cluster, $\lim _{\mathrm{s} \rightarrow \infty} \mathrm{M}_{\mathrm{s}}=\infty$. Therefore, for sufficiently large $\mathrm{s}>0 \mathbf{P}\left[\mathrm{M}_{\mathrm{s}}>(\mathrm{r} / \delta)\right]$ $>1-\varepsilon$. Note that $\left\{\mathrm{M}_{\mathrm{s}}>(\mathrm{r} / \delta)\right\}$ implies $\mathrm{t}\left(\gamma^{\prime}\right) \geq \mathrm{M}_{\mathrm{s}} \delta>\mathrm{r}$ for any $\gamma^{\prime}$ connecting $\mathrm{x}$ to $\mathrm{y}$ which touches $\partial \mathrm{B}_{\mathrm{s}}(\mathrm{X})$. Hence

$$
\mathbf{P}\left[\left\{\mathrm{t}\left(\gamma^{\prime}\right)>\mathrm{r} \text { : for any } \gamma^{\prime} \text { connecting } \mathrm{x} \text { to } \mathrm{y} \text { by touching } \partial \mathrm{B}_{\mathrm{s}}\right\}\right]>1-\varepsilon \text {. }
$$

Hence by combining (30) and (32), with probability greater than $1-2 \varepsilon$ the number of self-avoiding paths with the end sites $x$ and $y$ in $\mathbf{L}$ with passage time less than $r$ is finite (at least one). Therefore, it follows from the continuity of the distribution of the $t_{b}$ that with probability greater than $1-2 \varepsilon$, there exists a unique finite $\mathbf{L}$-geodesic connecting $\mathrm{x}$ to $\mathrm{y}$. Since $\varepsilon>0$ is arbitrary, the proof is complete.

The following is a large deviation estimate for the geometric length (the number of bonds) of the finite geodesic connecting two sites, used in the proof of Proposition 7. An analogous estimate was used in [8].

Lemma 9. Let $\mathbf{E}\left[\mathrm{t}_{\mathrm{b}}\right]<\infty$. Consider a half-plane

$$
\mathbf{L}=\left\{\left(\mathrm{x}_{1}, \mathrm{x}_{2}\right) \in \mathbf{Z}^{2}: \mathrm{h}_{1} \mathrm{x}_{1}+\mathrm{h}_{2} \mathrm{x}_{2} \geq \mathrm{n}\right\} .
$$

There exists a $c>0$ such that for any a $>0$ there exists an $L$ for which, whenever $x, y \in \mathbf{L}$ and $|x-y| \geq L$,

$$
\mathbf{P}\left[\left|\gamma_{\mathbf{L}}(\mathrm{x}, \mathrm{y})\right| \geq \mathrm{c}|\mathrm{x}-\mathrm{y}|\right] \leq \exp (-|\mathrm{x}-\mathrm{y}|)+\mathrm{a} .
$$

Proof. Let $b>\mathbf{E}\left(t_{b}\right)$ and $\lambda>0$ be such that $4 \mathbf{E} \exp \left(-\lambda t_{b}\right)<\frac{1}{2}$. Take $a$ constant $c$ such that $4 \mathrm{e}^{\lambda \mathrm{b} / \mathrm{C}} \operatorname{E} \exp \left(-\lambda \mathrm{t}_{\mathrm{b}}\right) \leq \frac{1}{2}$ and $\left(\frac{1}{2}\right)^{\mathrm{c}-1} \leq \exp (-1)$.

By the weak law of large numbers for independent random variables, for any $a>0$ there exists a constant $L>0$ such that for all pairs of $x$ and $y$ in $\mathbf{L}$ and $|x-y| \geq L$,

$$
\mathbf{P}\left[t_{L}(x, y) \geq b|x-y|\right] \leq a \text {. }
$$


We have

$$
\begin{aligned}
& \mathbf{P}\left[\left|\gamma_{\mathbf{L}}(\mathrm{x}, \mathrm{y})\right| \geq \mathrm{c}|\mathrm{x}-\mathrm{y}|, \mathrm{t}_{\mathbf{L}}(\mathrm{x}, \mathrm{y}) \leq \mathrm{b}|\mathrm{x}-\mathrm{y}|\right] \\
& \leq \sum_{[\gamma: x \rightarrow y,|\gamma| \geq c|x-y|]} \mathbf{P}[\mathrm{t}(\gamma) \leq \mathrm{b}|\mathrm{x}-\mathrm{y}|] \\
& \leq \sum_{[n \geq c|x-y|]} \sum_{[|\gamma|=n, \gamma: x \rightarrow y]} \mathbf{P}[t(\gamma) \leq b|x-y|] \\
& \leq \sum_{[n \geq c|x-y|]} \sum_{[|\gamma|=n, \gamma: x \rightarrow y]} \mathbf{E}[\exp \lambda(b|x-y|-t(\gamma))] \\
& \leq \sum_{n \geq c|x-y|} 4^{n} \exp (\lambda b|x-y|)\left[E \exp \left(-\lambda t_{b}\right)\right]^{n} \\
& \leq \sum_{n \geq c|x-y|}\left(\frac{1}{2}\right)^{n} \leq\left(\frac{1}{2}\right)^{c|x-y|-1} \leq \exp (-|x-y|)
\end{aligned}
$$

By combining (34) and (35), the proof is complete.

REMARK. If the variables $t_{b}$ have a finite exponential moment $E\left[e^{\varepsilon t_{b}}\right]$ for some $\varepsilon>0$, the right-hand side of (33) can be replaced by a more familiar expression $\exp (-|x-y|)$. We do not need this stronger estimate.

\title{
REFERENCES
}

[1] Kesten, H. (1986). Aspects of first-passage percolation. École d'Été de Probabilities de Saint-Flour XIV. Lecture Notes in Math. 1180 125-264. Springer, Berlin.

[2] Kesten, H. (1987). Percolation theory and first-passage percolation. Ann. Probab. 15 1231-1271.

[3] Kesten, H. (1993). On the speed of convergence in first-passage percolation. Ann. Appl. Probab. 3 296-338.

[4] Kesten, H. (1987). Surfaces with minimal random weights and maximal flows: a higher dimensional version of first-passage percolation. Illinois J . Math. 31 99-166.

[5] LiceA, C. and Newman, C. M. (1995). Geodesics in two-dimensional percolation. Ann. Probab. 24 399-410.

[6] Newman, C. M. (1979). Topics in Disordered Systems. Birkhäuser, Basel. To appear.

[7] Newman, C. M. (1995). A surface view of first-passage percolation. In Proceedings of the 1995 International Congress on Mathematics (S. D. Chatterji, ed.) 1017-1023. Birkhäuser, Boston.

[8] WeHR, J. (1997). On the number of infinite geodesics and ground states in disordered systems. J . Statist. Phys. To appear.

\author{
Department of MATHEMATics \\ and Program in Applied Mathematics \\ UNIVERSITY OF ARIZONA \\ TUCSON, ARIZONA 85721 \\ E-MAIL: wehr@math.arizona.edu \\ jwoo@math.arizona.edu
}

